\title{
Robust control strategy for improving the performance of a soft robotic link
}

\author{
Luis Nagua, Jorge Muñoz, Lisbeth Mena, Concepción A. Monje, and Carlos Balaguer \\ Systems Enginering and Automation Department, RoboticsLab \\ University Carlos III of Madrid \\ Madrid, Spain \\ lnagua@ing.uc3m.es, jmyanezb.ing@uc3m.es, lmena@pa.uc3m.es, cmonje@ing.uc3m.es, balaguer@ing.uc3m.es
}

\begin{abstract}
The robotic neck mechanism considered in this paper has as main element a soft link that emulates a human neck with two DOF (flexion, extension and lateral bending). The mechanism is based on a Cable-Driven Parallel Mechanism (CDPM) with components easy to manufacture in a $3 D$ printer. Due to the soft link properties and the platform mechanics, it is important to provide a robust control system. Two designs, a robust PID controller and a Fractional Order PI controller (FOPI) are proposed and compared, the fractional order control showing an enhanced performance. Both control approaches are tested in the real prototype, validating the soft neck feasibility and showing the robustness of the platform to mass changes at the neck tip.
\end{abstract}

Keywords: Soft robotic neck, Cable-Driven Parallel Mechanism (CDPM), Fractional order control, Robust control.

\section{Introduction}

In the case of humanoid robotics, a robot with soft links has the following main advantages: a) simplicity of design, favoring an under-actuated architecture; b) accessibility and adaptability to complex environments; and c) safer interaction with the human and the environment.

There are several humanoid neck mechanisms developed by different researchers. They can be divided into two categories: serial necks and parallel necks. The neck in series is very used due to its easy control, since each DOF is operated independently. For instance, HRP-4 [1] and Honda ASIMO-2002 [2] have two DOF (pitch, yaw).

Parallel robot necks are based in general on a parallel manipulator, which consists of a mobile platform, a fixed base, several identical active chains and a passive backbone, if necessary. This type of mechanism is interesting for the following reasons: the number of actuators is minimal; the number of sensors necessary for the closed-loop control of the mechanism is also minimal; when the actuators are locked, the manipulator remains in its position, which is an important safety aspect for certain applications, such as medical robotics [3], where they design a micro-robot for intestinal inspection which uses pneumatic actuators in its bendable portion.

In this paper we present a soft link to be used interchangeably in various limbs of the humanoid robot, like neck and spine, under the constraints of scalability, controllability of its stiffness and integration. The first step towards this goal is the design and performance analysis of a prototype of soft link working as a neck, studying its mathematical model and proposing a control system that guarantees the performance robustness to mass changes at the tip. The proposed soft neck simulates the structure of the human neck, where the cables simulate the muscles and tendons and the flexible link the spinal column. All this in a configuration of parallel mechanism.

Due to the neck design and its elastic behavior, the actuators will have different load torques at every different position, going through a varying load path for every movement. Therefore, a robust controller is necessary, and a Fractional Order Control approach is considered and compared to a robust PID controller design.

The most common Fractional Order Controllers (FOC) are Fractional Proportional Integral Derivative $\left(\mathrm{PI}^{\lambda} \mathrm{D}^{\mu}\right)$ ones, used for the first time by Podlubny [4] and further developed in works such as [5], [6], or [7].

The usual approach, specially for motion control, is to use a Fractional Derivative control, leaving the integrator out. This control scheme has the advantage of using the position sensor as an integrator, which simplifies the controller, while the steady state error is still cancelled, making the integral part unnecessary, as in [8], and later in [9] or [10]. Our situation is different, since the different neck inclinations will mean a variable system perturbation that needs to be addressed in order to cancel the stationary error. This will lead to choose a controller with integral part, as in similar approaches [11], [12] and [13].

In order to tune the controller, different meth- 
ods are available. Many of them are based on the numeric solution of nonlinear equations systems [7][12][10]. Others are based on optimization methods, such as Particle Swarm Optimization algorithm (PSO) [6][13], Artificial Bee Colony algorithm (ABC) [14][15], Firefly Algorithm (FA) [16], or Differential Evolution method (DE) [17]. See [18] for a comparative study of optimization algorithms applied to FOC.

We will tune the proposed FOC using Monje's Method as described in [5]. The PID controller will be also tuned following this approach, but modified in order to allow its application to integer order controllers.

The paper is organized as follows. Section II introduces the soft neck proposal and its mathematical model. Section III presents the control problem and proposes two controllers that grant the robustness of the system to mass changes at the neck tip. Section IV discusses the results obtained from the control of the neck using a PID and a FOC. Finally, Section V outlines the main conclusions of the work.

\section{Robotic Neck}

The design of this neck is based on the configuration of a parallel robot according to the advantages mentioned in [19]. Fig. 1 shows the different motions that the human neck can perform, together with the range of movement associated to each motion. This Figure has been extracted from [20]. As can be appreciated, the human neck has three main motions: rotation, flexion and extension, and lateral bend.
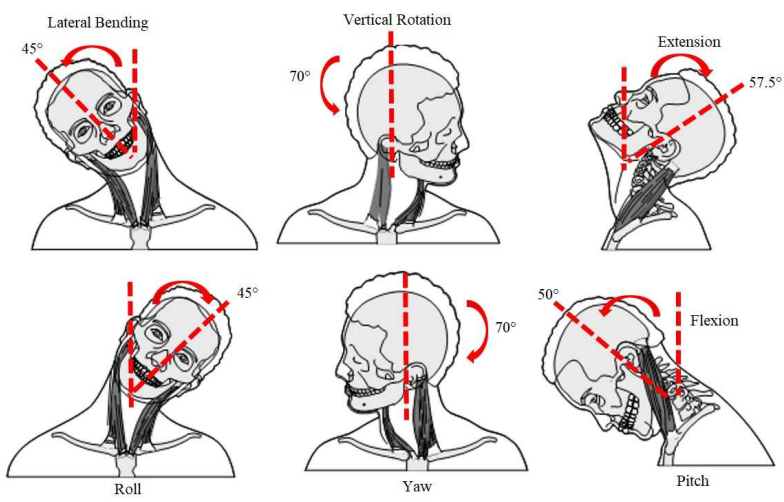

Figure 1: Human neck motions.

The constraints for the design of our soft robotic neck are the following: two Degrees of Freedom (DOF) with a maximum tilt angle of $40^{\circ}$, under a criterion of flexibility and inspired by the human neck structure; it uses a soft link as neck; the mechanism supports different loads at the neck tip.
The CAD design of the neck is shown in Fig. 2, and its equivalent schematic representation in Fig. 3 .

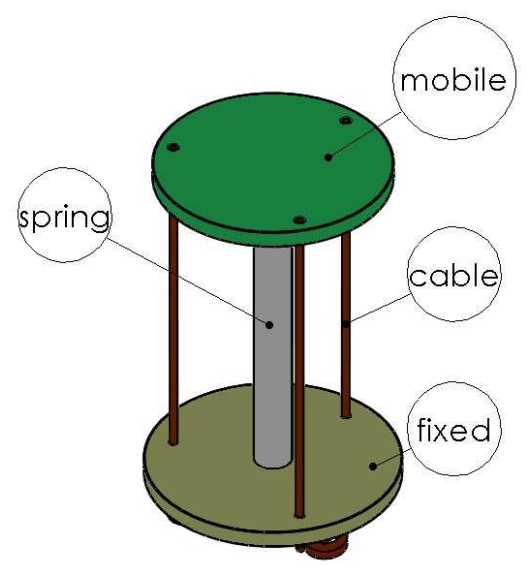

Figure 2: Prototype functional scheme.

The soft neck shown in Fig. 3(a) consists of: a fixed base, a moving platform, three flexible cables with negligible mass and a soft link made of flexible Thermoplastic Elastomer (TPE) material. The moving platform is driven by three cables and the connection points are $\vec{B}=\left(B_{1} ; B_{2} ; B_{3}\right)$; the other end of each cable connects to a roller driven by a motor and the cables pass through the fixed base at points $\overrightarrow{O A}=\left(A_{1} ; A_{2} ; A_{3}\right)$. We will denote the cable length between $A_{i}$ and $B_{i}$ as $L_{i}$.

There are two independent parameters: angle $\theta_{p}$ between the fixed base plane and the moving platform plane (tilt angle); and angle $\theta_{s}$ between axis $s$ and axis $X$ (orientation angle). $s_{o}$ and $t_{0}$ are dependent parameters, and once given values to the independent parameters, $s_{o}$ can be solved.

The homogeneous transformation matrix (1) represents the projection from frame oxyz to $O X Y Z$.

$$
{ }^{O} T_{o^{\prime}}=\left[\begin{array}{cc}
{ }^{O} R_{o^{\prime}} & P_{o} \\
0 & 1
\end{array}\right]
$$

where $P_{o}$ is the position vector of point $o$ with respect to the base coordinate frame, $P_{o}=$ $\left[\begin{array}{ccc}s_{o} \cos \theta_{s} & s_{o} \sin \theta_{s} & t_{0}\end{array}\right]^{T}$, and ${ }^{O} R_{o^{\prime}}\left(\theta_{s}, \theta_{p}\right)$ is the rotational matrix that describes the orientation of the moving platform using the Euler angles with orientation $Z Y Z$.

The inverse position kinematics problem is to calculate the cable lengths $L=\left[\begin{array}{lll}L_{1}, & L_{2}, & L_{3}\end{array}\right]^{T}$ given the desired moving platform posture $x=\left[\begin{array}{ll}\theta_{s} & \theta_{p}\end{array}\right]^{T}$.

The cable lengths are calculated by: 


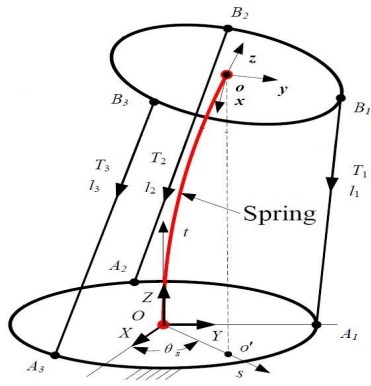

(a)

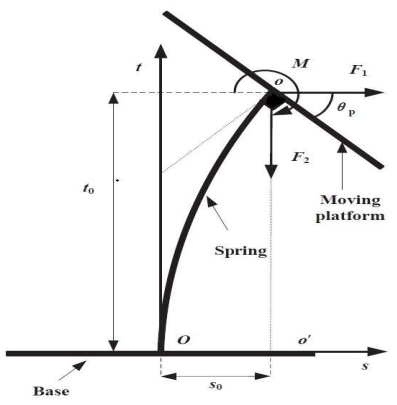

(b)

Figure 3: Robot neck diagrams

(a) CDMP model. (b) Lateral bending of the neck.

$$
L_{i}=\left\|{ }^{O} T_{o^{\prime}} \overrightarrow{o B_{i}}-\overrightarrow{O A_{i}}\right\| \quad(i=1,2,3)
$$

However, $s_{o}$ and $t_{0}$ cannot be arbitrary numbers. In this case, $s_{o}$ and $t_{0}$ will be resolved geometrically, considering the flexion of the soft link (see Fig. $3(b)$ ) as an arc with curvature radius

$$
R=\frac{L_{o}}{\theta_{p}}
$$

where $L_{o}$ is the length of soft link. Based on triangle geometries, one has

$$
R=s_{o}+R \cos \theta_{p}
$$

and equalizing equations (3) and (4), the result is

$$
s_{o}=\frac{L_{o}\left(1-\cos \theta_{p}\right)}{\theta_{p}}
$$

and $t_{0}$ is given by

$$
t_{o}=\frac{L_{o} \sin \theta_{p}}{\theta_{p}}
$$

whose constants values are given in Table 1 .

The implementation is performed varying $\theta_{p}$ from $0^{\circ}$ to $40^{\circ}$ and $\theta_{s}$ from $0^{\circ}$ to $360^{\circ}$. The results of the inverse position kinematics are shown in Fig. 4, where the cable lengths are located in the z-axis.
Table 1: PARAMETERS OF SELECTED COMPRESSIVE SPRING

\begin{tabular}{|c|c|c|c|c|}
\hline$L_{o}[\mathrm{~m}]$ & $\mid \overrightarrow{O A_{i}}$ & {$[\mathrm{~m}]$} & $\mid \overrightarrow{{ }_{B}}$ & {$[\mathrm{~m}]$} \\
\hline 0.107 & 0.05 & 0.05 \\
\hline
\end{tabular}

As shown in Fig. 4, for large values of $\theta_{p}$, the variation in cable length is also large. This is because the more the mobile base is tilted, the greater amount of force required.
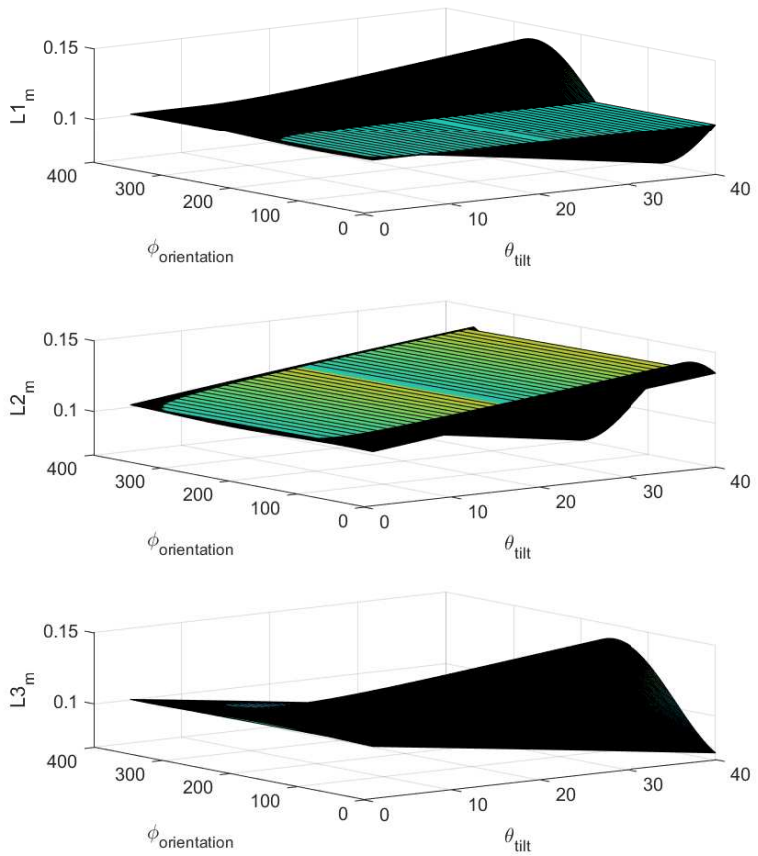

Figure 4: Inverse position kinematics.

\section{Control Problem}

The control problem, represented in Fig. 5, focuses on the motors driving the neck tendons. The platform target positions are defined by their orientation and inclination $\left(\theta_{s}\right.$ and $\theta_{p}$, respectively). Solving the inverse kinematics as described in the previous section, the lengths for each cable are obtained. Due to the transmission mechanism, each cable length corresponds to a specific motor angular position; therefore, motor position control is needed to achieve and hold the platform target positions.

In order to achieve each required motor position $\theta_{i}$, a feedback control loop configuration is used (output position, velocity actuated). The neck prototype developed for this purpose is equipped with encoder position sensors, and each motor receives velocity commands in rpm. The angular position $\theta_{m}$ measured by the encoder is compared 
to the reference angular position $\theta_{i}$ resulting from the application of the inverse kinematics, and the controller will then be in charge of commanding the servomotors in velocity.

The motor model obtained from the experimental identification process using velocity input and position output, results in:

$$
G(s)=\frac{54.89}{54.89+s} \cdot \frac{k_{e n c}}{s},
$$

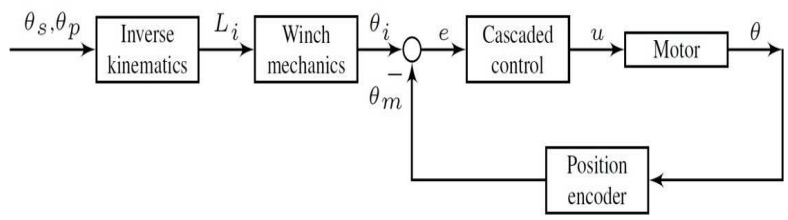

Figure 5: Structure and interfaces of the soft neck control scheme

where $k_{\text {enc }}=6$ is a known gain introduced by the encoder.

The time and frequency responses of the motor model are shown in Fig.6. Note that phase margin $\phi_{m}=83.8 \mathrm{deg}$ at the gain crossover frequency $\omega_{c}=5.965 \mathrm{rad} / \mathrm{s}$.
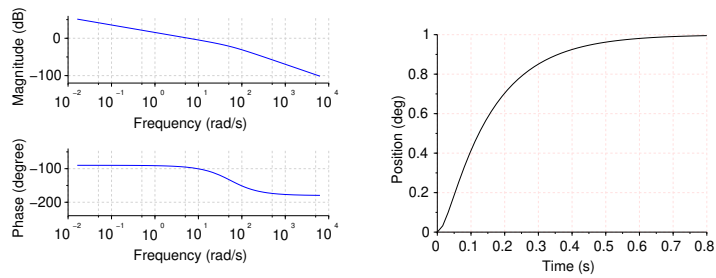

Figure 6: Bode diagram (left) and feedback unit step response (right) for the motor model.

In order to tune the controllers, phase margin $\phi_{m}$ and gain crossover frequency $\omega_{c}$ specifications will be used. Having an integrator in the plant, it is common practice to avoid the integrator in the controller, leading to a proportional derivative approach; but in this case, because of the elastic nature of the system, the tendons will need different forces at the different positions, resulting in a variable motor torque needed for the different neck inclinations, making it necessary to use an integral controller for stationary error cancellation. In addition, the neck will accept different payloads attached to the tip, which adds uncertainties to the model and asks for a robust control system.

According to this, the control specifications to be met have been selected according to Monje's
Method [5], which considers the following constraints:

- Phase margin $\phi_{m}$ and gain crossover frequency $\omega_{c}$

- Robustness to mass changes on the tip at a nominal crossover frequency $\omega_{c}$

- Robustness to model uncertainties

From the basic definitions of phase margin $\phi_{m}$ and gain crossover frequency $\omega_{c}[21]$, the following two equations are obtained:

$$
\begin{gathered}
0=\arg \left[G\left(j \omega_{c}\right) C\left(j \omega_{c}\right)\right]+\pi-\phi_{m} \\
0=\left|G\left(j \omega_{c}\right) C\left(j \omega_{c}\right)\right|-1
\end{gathered}
$$

In order to achieve control robustness, another specification is normally considered. First stated by Hendrik Bode, and later in works such as [12], $[22],[5],[10]$, open loop flat phase specification is another common constraint in controller design, as demonstrated in [23]. In our system, the model gain is affected by the payloads of the device, as proved in [24]. Therefore, meeting this specification will make the system robust to changes in the payload configuration. The formulation for this constraint is shown in the following equation (see $[5])$ :

$$
\left(\frac{d\left(\arg \left(C\left(j \omega_{c g}\right) G\left(j \omega_{c g}\right)\right)\right)}{d \omega}\right)_{\omega=\omega_{c g}}=0 .
$$

In order to validate the platform and compare the results, two controllers will be designed: a robust PID controller and a Fractional Order Proportional Integral (FOPI) controller, both based on the fulfillment of the control specifications previously defined. In the next sections, the design of these controllers will be detailed.

\subsection{PID Control}

The PID controller formula can be stated as follows:

$$
C(s)=k_{p}+k_{i} \frac{1}{s}+k_{d} s
$$

with $k_{p}, k_{i}$ and $k_{d}$ the proportional, integral and derivative gains, respectively.

The open loop system is given by: 


$$
C(s) G(s)=\frac{k_{p}+k_{i} \frac{1}{s}+k_{d} s}{1+\frac{1}{54.89} s} \cdot \frac{k_{e n c}}{s}
$$

To find the values of $k_{p}, k_{i}$ and $k_{d}$ that meet the specifications, equations (8), (9) and (10) need to be solved. Using Scilab fsolve function, and considering $\omega_{c}=20 \mathrm{rad} / \mathrm{s}$ and $\phi_{m}=45 \mathrm{deg}$, the resulting controller parameters are $k_{p}=3.216$, $k_{i}=29.967$ and $k_{d}=0.0000061$. The Bode diagram of the open loop system with this controller and the step response are shown in Fig. 7, where it is clearly seen that the specifications are fulfilled.

The specifications of $\omega_{c}$ and $\phi_{m}$ have been set to those given values in order for the system to have a fast response (related to the value of $\omega_{c}$ ) and a certain overshoot (related to the value of $\phi_{m}$ ). The higher the gain crossover frequency, the higher the system speed; the lower the phase margin, the higher the overshoot. With $\phi_{m}=45 \mathrm{deg}$ we can guarantee a certain overshoot (underdamped system, as shown in Fig. 7) and the robustness to mass changes can be more appropriately studied.
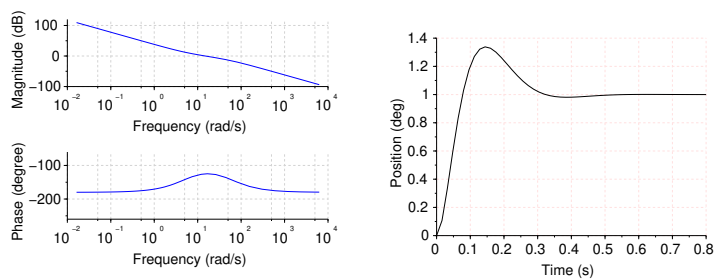

Figure 7: Bode diagram (left) and step response (right) for the PID control system

\section{$3.2 \quad$ Fractional Order Control}

Here we propose a FOC described by (13):

$$
C(s)=k_{p}+k_{a} s^{\alpha}
$$

where $k_{p}$ is the proportional gain, $k_{a}$ is the fractional gain, and $\alpha$ is the fractional order. According to the sign of $\alpha$, the resulting controller can have a fractional order derivative component (positive $\alpha$ ) or an fractional order integral component (negative $\alpha$ ).

Having three parameters available, the specifications can be fulfilled, granting the system robustness to gain changes. The open loop system is modeled by the following equation:

$$
C(s) G(s)=\frac{k_{p}+k_{a} s^{\alpha}}{1+\frac{1}{54.89} s} \cdot \frac{k_{e n c}}{s}
$$

Again, solving the nonlinear equations system using Scilab fsolve function, we can find the values of $k_{p}, k_{a}$ and $\alpha$ that meet the specifications stated in equations (8), (9) and (10). Considering the same $\omega_{c}=20 \mathrm{rad} / \mathrm{s}$ and $\phi_{m}=45 \mathrm{deg}$ performance specifications, the resulting controller parameters are $k_{p}=2.99, k_{a}=22.744$ and $\alpha=-0.9$. Having a negative fractional order, the resulting controller is a Fractional Order Proportional Integrator (FOPI).

The Bode diagram of the open loop system with this controller and its closed loop step response are shown in Fig. 8. A flat phase slope at the required gain crossover frequency can be seen in the figure, which also shows that the phase margin requirement is met.

Another asset of the FOPI is the following. The phase margin tends to $\phi_{m}=0 \mathrm{deg}$ for varying gains in the case of the PID control system, as can be seen in the Bode diagram of Fig. 7, which affects the stability of the system very negatively. However, the fractional order $\alpha$ of the integrator in the case of the FOPI, when $\alpha_{j} 1$ (our particular case), guarantees that the controller's phase is $\phi_{c}>-90 \mathrm{deg}$, since the contribution in phase of the FO integrator is $-\alpha \cdot 90 \mathrm{deg} * 90 \mathrm{deg}$. This fact enhances the system stability for varying gains, since the phase margin will never reach zero value.
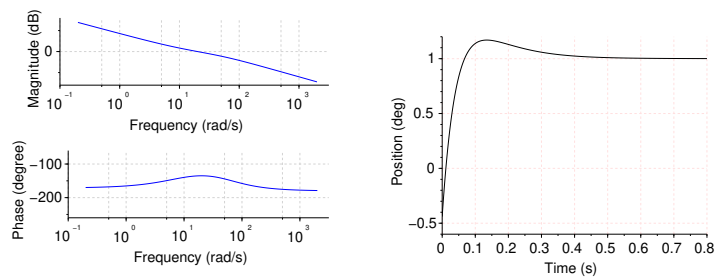

Figure 8: Bode diagram (left) and step response (right) for the FOPI control system

\section{Experimental results}

The experiments were designed to test both controllers robustness and performance. In that sense, different masses were attached to the neck tip, which, together with the different neck inclinations, introduces actuators gain variations in the system and makes this case study very suitable to study controllers robustness to model uncertainties. The different masses attached to the upper platform were $m=(0 g, 200 g, 400 g, 600 g$, $800 \mathrm{~g}, 1000 \mathrm{~g}$ ), and the neck experiments were carried out with the PID and FOPI control systems.

The results from the experiments show that both systems present good robustness and performance, as illustrated in Fig. 9. However, it is clear that the PID control strategy presents bigger overshoot variations for the different masses than the FOPI 
control approach. Besides, the stability is worse in general terms in the case of the integer order controller, which shows that the introduction of the fractional order $\alpha$ in the controller improves system stability.
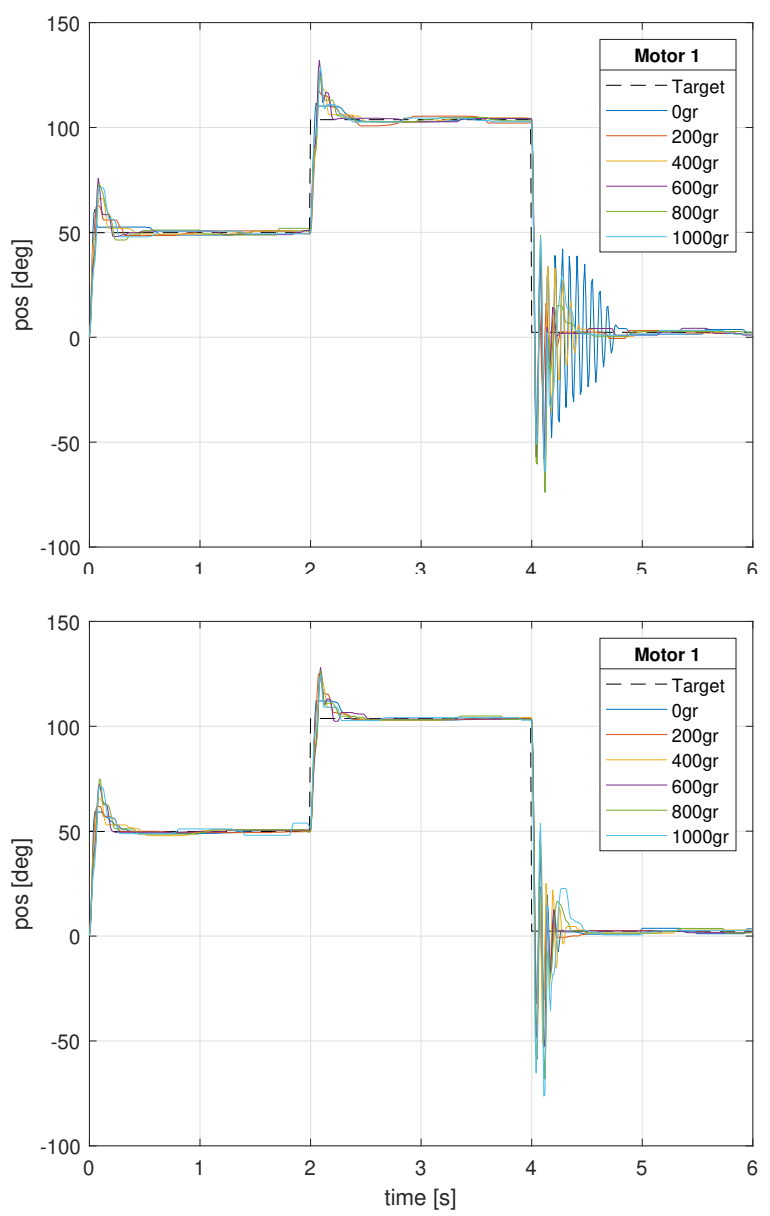

Figure 9: Step responses of the two proposed controllers, PID (top) and FOPI (bottom), for different masses located at the tip. Step inputs of 50deg and $-100 \mathrm{deg}$.

As can be observed in Fig. 10, the system stability is very poor for the PID controller in the reverse trajectory, when the neck returns to the initial position. Under this condition, the neck elastic action combined with the mass inertia reduces the motor load torque to a value close to zero, increasing the system gain and consequently the crossover frequency. Due to the tiny phase margin at high frequencies for the PID controller (see Fig. 7), this fact will decrease system stability. In fact, during the experiments with the PID controller and for certain position references, the amplitude of oscillation reached a high value that triggered the driver protections and switched the system into an error state, reason why the target position had to be redefined.

This situation is greatly mitigated by the use of the fractional order controller because of its effect on the phase margin. The FOC exponent (0.9 in our case) makes the controller phase to be $\phi_{c}>$ -90 , which results in an open loop phase margin $\phi_{m}>0$ for whatever gain crossover frequency, as shown in Fig. 8. This is contrary to what happens with the integer order controller, where the phase margin tends to zero as the gain increases, making the system less stable.

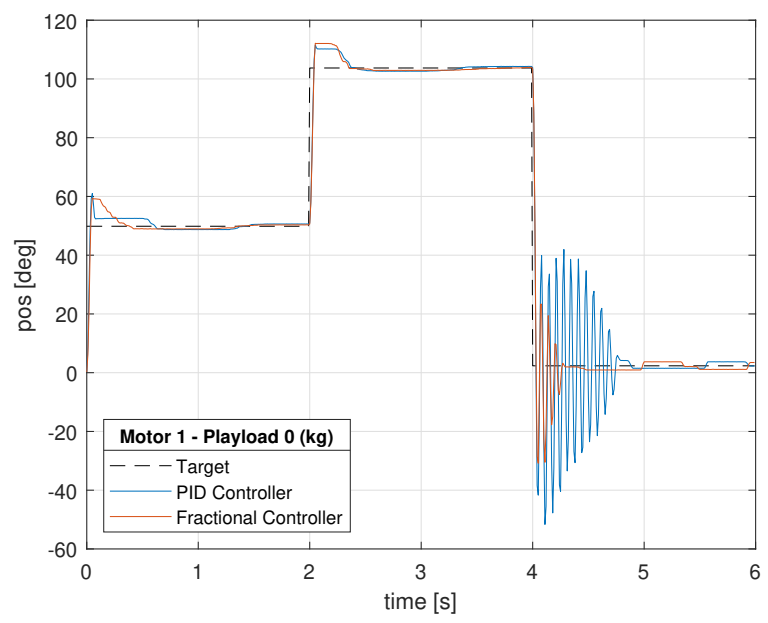

Figure 10: Comparison of PID and FOPI control systems for the case of $0 g$ payload. Step inputs of $50 \mathrm{deg}$ and $-100 \mathrm{deg}$.

The performance of the system with both controllers is show in the video at: link.

\section{Conclusions}

This paper has presented the design of a soft robotic neck with two DOF providing pitch and roll movements. The movement constraint is a maximum inclination of $40^{\circ}$. The electromechanical design of the prototype have been addressed, which uses a coil compression spring to simulate the cervical vertebrae and cables that act as muscles. The mathematical model of the neck has been presented, including its inverse kinematics.

Two methods have been applied for the control of the soft robotic neck: a standard PID control approach and a Fractional Order PI (FOPI) one. The results validate both controllers for their use in the soft neck platform; however, due to the neck inherent varying gain property, the FOPI control system presents a better performance, showing a more enhanced robustness and stability for the entire actuation range when compared with the PID control alternative. 


\section{Acknowledgement}

The research leading to these results has received funding from the project Desarrollo de articulaciones blandas para aplicaciones robóticas, with reference IND2020/IND-1739, funded by the Comunidad Autónoma de Madrid (CAM) (Department of Education and Research), and from RoboCity2030-DIH-CM, Madrid Robotics Digital Innovation Hub (Robótica aplicada a la mejora de la calidad de vida de los ciudadanos, FaseIV; S2018/NMT-4331), funded by "Programas de Actividades I+D en la Comunidad de Madrid" and cofunded by Structural Funds of the EU.

\section{References}

[1] H. Hirukawa, F. Kanehiro, K. Kaneko, S. Kajita, K. Fujiwara, Y. Kawai, F. Tomita, S. Hirai, K. Tanie, T. Isozumi et al., "Humanoid robotics platforms developed in HRP," Robotics and Autonomous Systems, vol. 48, no. 4, pp. 165-175, 2004.

[2] Y. Sakagami, R. Watanabe, C. Aoyama, S. Matsunaga, N. Higaki, and K. Fujimura, "The intelligent asimo: System overview and integration," in IEEE/RSJ international conference on intelligent robots and systems, vol. 3. IEEE, 2002, pp. 2478-2483.

[3] G. Thomann, M. Bétemps, and T. Redarce, "Study of a new intelligent tool for the colonoscopy," CMBES Proceedings, vol. 27, 2002.

[4] I. Podlubny, "Fractional-order systems and $\mathrm{pi}^{\lambda} \mathrm{d}^{\mu}$-controllers," IEEE Transactions on Automatic Control, vol. 44, no. 1, pp. 208214, Jan 1999.

[5] C. A. Monje, B. M. Vinagre, V. Feliu, and Y. Chen, "Tuning and auto-tuning of fractional order controllers for industry applications," Control engineering practice, vol. 16, no. 7, pp. 798-812, 2008.

[6] A. Rastogi and P. Tiwari, "Optimal tuning of fractional order pid controller for dc motor speed control using particle swarm optimization," International Journal of Soft Computing and Engineering (IJSCE), ISSN, pp. 2231-2307, 2013.

[7] K. Ranjbaran and M. Tabatabaei, "Fractional order [pi], [pd] and [pi][pd] controller design using bode's integrals," International Journal of Dynamics and Control, vol. 6, no. 1, pp. 200-212, Mar 2018.
[8] L. Dorcak, "Numerical models for the simulation of the fractional-order control systems," arXiv preprint math/0204108, 2002.

[9] M. F. Silva and J. T. Machado, "Fractional order $\mathrm{pd}^{\alpha}$ joint control of legged robots," Journal of Vibration and Control, vol. 12, no. 12, pp. 1483-1501, 2006.

[10] T. Qingshun, W. Chunfu, Y. Yuanhui, L. Guodong, and Z. Fengyu, "Design and implementation of fractional order controller for service robots," International Journal of Control and Automation, vol. 8, no. 5, pp. 209-220, 2015.

[11] C. A. Monje, Y. Chen, B. M. Vinagre, D. Xue, and V. Feliu, Fractional-order Systems and Controls: Fundamentals and Applications. Springer, 2010.

[12] I. Petráš, "Fractional-order feedback control of a dc motor," Journal of electrical engineering, vol. 60, no. 3, pp. 117-128, 2009.

[13] K. Khandani and A. A. Jalali, "Robust fractional order control of a DC motor based on particle swarm optimization," in $M E M S$, NANO and Smart Systems, ser. Advanced Materials Research, vol. 403. Trans Tech Publications, 2 2012, pp. 5030-5037.

[14] D. Karaboga and B. Basturk, "A powerful and efficient algorithm for numerical function optimization: artificial bee colony (abc) algorithm," Journal of global optimization, vol. 39, no. 3, pp. 459-471, 2007.

[15] A. Rajasekhar, P. Kunathi, A. Abraham, and M. Pant, "Fractinal order speed control of dc motor using levy mutated artificial bee colony algorithm," in 2011 World Congress on Information and Communication Technologies, Dec 2011, pp. 7-13.

[16] V. H. Haji and C. A. Monje, "Fractionalorder PID control of a chopper-fed DC motor drive using a novel firefly algorithm with dynamic control mechanism," Soft Computing, vol. 22, no. 18, pp. 6135-6146, 2018.

[17] F. Martín, C. A. Monje, L. Moreno, and C. Balaguer, "DE-based tuning of $\mathrm{pi}^{\lambda} \mathrm{d}^{\mu}$ controllers," ISA Transactions, vol. 59, pp. 398 $-407,2015$.

[18] S. Al-Ratrout, A. Saleem, and H. Soliman, "Optimization methods in fractional order control of electric drives: A comparative study," in 2015 10th International Symposium on Mechatronics and its Applications (ISMA). IEEE, 2015, pp. 1-6. 
[19] S. Behzadipour and A. Khajepour, "Cablebased robot manipulators with translational degrees of freedom," in Industrial Robotics: Theory, Modelling and Control. InTech, 2006, pp. 221-236.

[20] L. Nagua, C. A. Monje, J. Muñoz, and C. Balaguer, "Design and performance validation of a cable-driven soft robotic neck," Spanish Robotics Conference, 2018.

[21] W. K. Ho, C. C. Hang, and L. S. Cao, "Tuning of PID controllers based on gain and phase margin specifications," Automatica, vol. 31, no. 3, pp. 497-502, 1995.

[22] C. Monje, F. Ramos, V. Feliu, and B. Vinagre, "Tip position control of a lightweight flexible manipulator using a fractional order controller," IET Control Theory \& Applications, vol. 1, no. 5, pp. 1451-1460, 2007.

[23] Y. Chen, C. Hu, and K. L. Moore, "Relay feedback tuning of robust PID controllers with iso-damping property," in 42nd IEEE International Conference on Decision and Control (IEEE Cat. No. 03CH37475), vol. 3. IEEE, 2003, pp. 2180-2185.

[24] V. Feliu, B. M. Vinagre, and C. A. Monje, "Fractional-order control of a flexible manipulator," in Advances in Fractional Calculus. Springer, 2007, pp. 449-462.

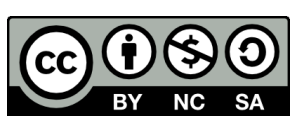

(C) 2021 by the authors. Submitted for possible open access publication under the terms and conditions of the Creative Commons Attribution CC BY-NC-SA 4.0 license (https://creativecommons.org/licenses/by-ncsa/4.0/deed.es). 